\title{
Numerical simulations of magnetic structures
}

\author{
I. N. Kitiashvili ${ }^{1,2}$, A. G. Kosovichev ${ }^{1}$, A. A. Wray ${ }^{1}$ and \\ N. N. Mansour ${ }^{3}$ \\ ${ }^{1}$ W.W. Hansen Experimental Physics Laboratory, Stanford University, \\ Stanford, CA 94305, USA \\ email: sasha@sun.stanford.edu \\ ${ }^{2}$ Center for Turbulence Research, Stanford University, \\ Stanford, CA 94305, USA \\ email: irinasun@stanford.edu \\ ${ }^{3}$ NASA Ames Research Center, \\ Moffett Field, Mountain View, CA 94040, USA \\ email: Alan.A.Wray@nasa.gov, Nagi.N.Mansour@nasa.gov
}

\begin{abstract}
We use 3D radiative MHD simulations of the upper turbulent convection layer for investigation of physical mechanisms of formation of magnetic structures on the Sun. The simulations include all essential physical processes, and are based of the LES (Large-Eddy Simulations) approach for describing the sub-grid scale turbulence. The simulation domain covers the top layer of the convection zone and the lower atmosphere. The results reveal a process of spontaneous formation of stable magnetic structures from an initially weak vertical magnetic field, uniformly distributed in the simulation domain. The process starts concentration of magnetic patches at the boundaries of granular cells, which are subsequently merged together into a stable large-scale structure by converging downdrafts below the surface. The resulting structure represents a compact concentration of strong magnetic field, reaching $6 \mathrm{kG}$ in the interior. It has a cluster-like internal structurization, and is maintained by strong downdrafts extending into the deep layers.
\end{abstract}

Keywords. Sun: magnetic fields, sunspots; methods: numerical

\section{Introduction}

The recent progress in the numerical modeling has made it possible to reproduce in simulations many observational effects in the quiet Sun region, active regions, magnetic flux emerging and whole magnetic structures (e.g., Stein \& Nordlund 2001, Jacoutot et al. 2008, Kitiashvili et al. 2009, Cheung et al. 2008, Stein et al. 2010, Rempel et al. 2009). However, most of the modeling has been done by setting up the initial conditions with already existing magnetic structures, e.g. a horizontal flux tube for the modeling of magnetic flux emerging, or a vertical flux tube with strong field for the sunspot/pore structures simulations. It seems that so far only one study succeeded in reproducing a spontaneous formation of a micropore-like magnetic structure from an initially uniform field in the turbulent convection of the Sun (Stein et al. 2003), and the lifetime of this structure was very short.

Here, we present results of the realistic MHD simulations that show a process of spontaneous formation of a stable pore-like magnetic structure in fully developed convection from an uniform magnetic field. For the simulations we used a $3 \mathrm{D}$ radiative MHD code, "SolarBox" (Jacoutot et al. 2008). The code is built for 3D simulations of 

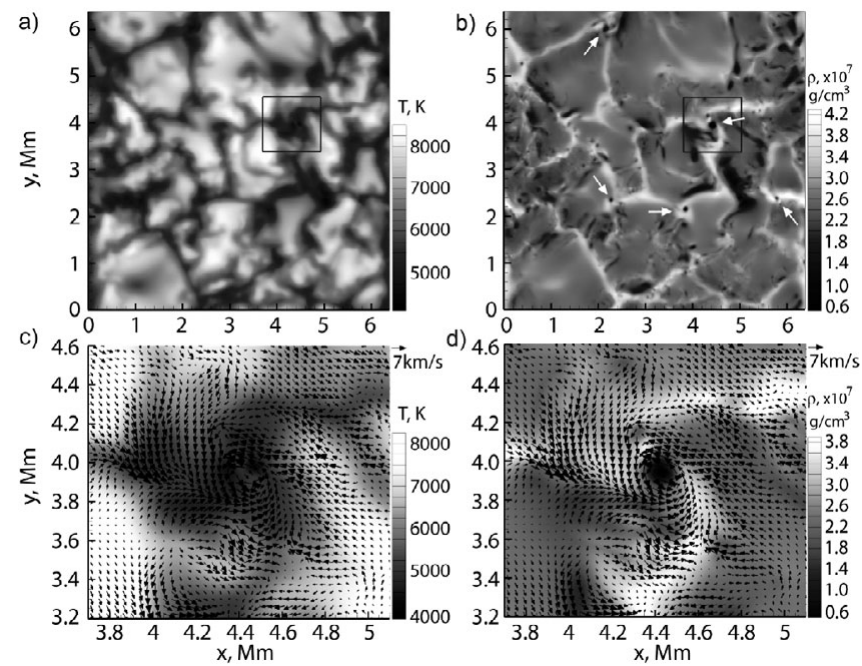

Figure 1. Snapshots of granular convection at the surface for the simulations without magnetic field, and the horizontal resolution of $12.5 \mathrm{~km}$ : temperature (left column) and density (right). The black square indicates a large whirlpool shown in more detail in panels c) - d). Black arrows show the flow velocity. White arrows in panel b) point to the centers of some vortices (dark low-density points).

compressible fluid flows in a magnetized and highly stratified medium of top layers of the convective zone and the low atmosphere, in the rectangular geometry. The simulation results are obtained for the computational domain of $6.4^{2} \times 5.5 \mathrm{Mm}$ with the grid sizes: $50^{2} \times 43 \mathrm{~km}, 25^{2} \times 21.7 \mathrm{~km}$ and $12.5^{2} \times 11 \mathrm{~km}\left(128^{2} \times 127,256^{2} \times 253\right.$ and $512^{2} \times 505 \mathrm{mesh}$ points). The domain includes a top, $5 \mathrm{Mm}$-deep, layer of the convective zone and the low atmosphere.

\section{Simulations of granulation and vortex tubes}

Initially we simulate the conditions of the quiet Sun in absence of magnetic field. Figure 1 shows snapshots for temperature (left column) and density (right) at the surface for the case without magnetic field. An interesting feature of the convective flows is the formation of whirlpool-like motions of different sizes $(\sim 0.2-1 \mathrm{Mm})$ and lifetimes ( 15-20 min at the vertexes of the intergranular lanes (Kitiashvili et al. 2010). The vortical motions are particularly well seen in the density variations. The centers of the whirlpools are seen as dark dots (indicated by white arrows in Fig. 1b) in the intergranular space. The evolution of these vortices (swirls) is ultimately related to the dynamics of convective motions in the domain. The convective flows sometime may collect the swirls in a local area, merge together, and then destroy them. Stronger vortices usually correlate with downflows, and this is also found in our results.

From time to time, convection creates pretty big whirlpools, as the one indicated by square in Fig. 1, which can swallow up other smaller swirls around them. The big swirls are usually easy to see also in the surface temperature and intensity variations. The detailed structure of a large whirlpool around a compact vortex tube with a sharp boundary is shown in Figs 1c-d. The whirlpool structure is characterized by (Kitiashvili et al. 2010): 1) formation of downdraft lanes (visible as "arms" in the Figs 1cd) of higher density that correlates with lower temperature; 2) a pronounced vortical 

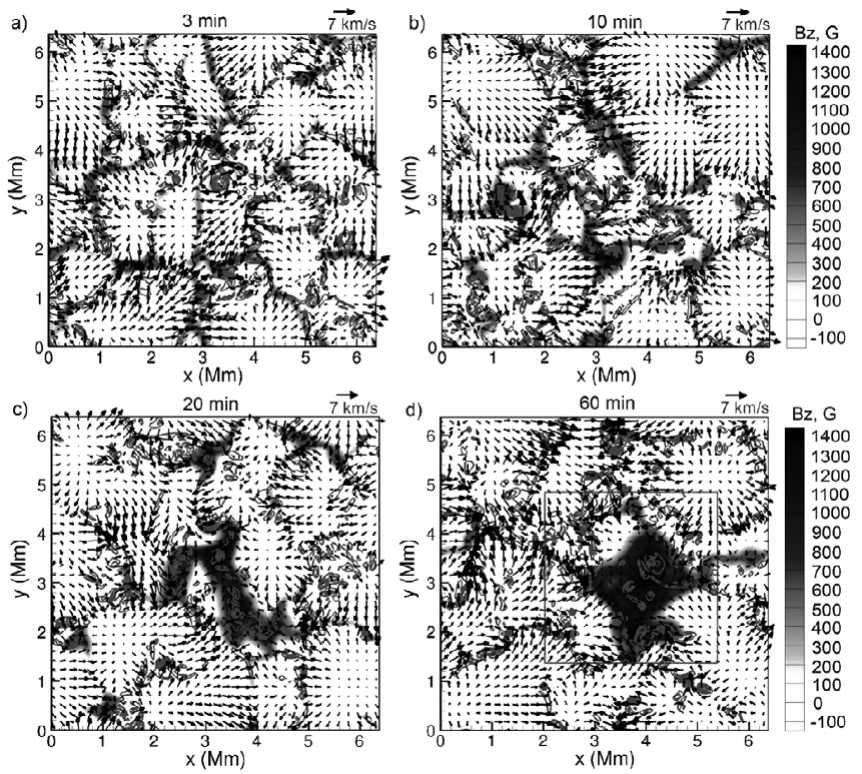

Figure 2. Snapshots of the surface distribution of vertical magnetic field (background), horizontal flows (arrows) and vorticity magnitude (contour lines) for four moments of time: 3,10 , 20 and $60 \mathrm{~min}$, from the moment of initiation of a uniform magnetic field $\left(B z_{0}=100 \mathrm{G}\right)$.

structure of the velocity flow; 3) increased magnitude of the horizontal velocity up to 7 $-9 \mathrm{~km} / \mathrm{s} ; 4)$ a sharply decreased density in the central core of the vortex, and a slightly higher temperature than in the surrounding. The typical depth of large swirls is about $100-200 \mathrm{~km}$. The vortical motions in the solar granulation have been detected in highresolution observations (e.g., Pötzi \& Brandt 2005, Bonet et al. 2008, 2010), and the observational results generally agree with the simulations.

\section{Spontaneous magnetic structure formation}

We made a series of simulations for the initial vertical uniform magnetic field, $B z_{0}=$ $100 \mathrm{G}$, different computational grids and domain sizes. Qualitatively the simulation results are very similar in all these cases, and show the formation of stable magnetic pore-like structures. In Figures 2 we present the results for the horizontal resolution of $25 \mathrm{~km}$, and the domain size of $6.4 \times 6.4 \times 5.5 \mathrm{Mm}$, for which we have made the longest run ( $\gtrsim 9$ hours). The periodic lateral boundary conditions allow us, for the illustration purpose, to shift the horizontal frame so that the structure is located close to the center. As we see in the simulations, the structure can be formed in any place of our computational domain, but usually the process starts at one of the strongest vortices (Kitiashvili et al. 2010).

Figure 2 shows snapshots of the vertical magnetic field (background image), horizontal flow field (arrows), and the vorticity magnitude (contour lines) for four moments of time: $3,10,20$ and $60 \mathrm{~min}$ after the moment $t=0$, when the $100 \mathrm{G}$ vertical field was uniformly distributed in the computational domain. During few minutes the magnetic field is swept into the intergranular lines, and is significantly amplified up to $\sim 500-1000 \mathrm{G}$. The vortices and magnetic field get concentrated at some locations in the intergranular lanes, where they are deformed and became elongated (or elliptically shaped) along the 
intergranular lines. The process of formation of a large-scale magnetic structure starts at a strongest vortex in our domain. The whirlwind causes deformation of the intergranular space, and creates a cavity of low density, temperature and pressure. The cavity expands and increases the accumulation of magnetic field (Fig. 2a). A similar process of magnetic field concentration, sweeping, twisting and stretching by vortical motions in the intergranular lane was initially observed in the simulations of Stein et al. (2003).

During the next few minutes the deformation of the "parent" vortex continues; then it gets destroyed on the surface by $t=10 \mathrm{~min}$ (Fig. 2b), but leaves strong downdraft motions in the interior. The process of accumulation of magnetic flux in this area continues. The local concentrations of magnetic field and vorticity get stronger and are moved by convective motions in the direction of the initial cavity, into the region where the gas pressure remains systematically low due to the downdrafts. The different small magnetic structures join together in a magnetic conglomerate that continues to attract other magnetic micro-structures (Fig. 2c) and becomes more compact (Fig. 2d).

In our simulations, the cluster structure is represented by internal field concentrations (flux tubes), $100-200 \mathrm{~km}$ thick, in which the field strength reaches $6 \mathrm{kG}$ after 1 hour (Kitiashvili et al. 2010). The velocity distribution shows strong, often supersonic, downflows around the magnetic structure. Inside the magnetic structure the convective flows are suppressed by strong magnetic field. However, despite the weak velocities $(\sim 0.1-$ $0.2 \mathrm{~km} / \mathrm{s}$ ) there are very small elongated convective cells resembling the umbral dots observed at the surface. We have followed the evolution of the magnetic pore-like structure for more than 8 solar hours, and did not see any indication of its decay. The structure continues to evolve, changes the shape and internal structurization, but remains compact.

\section{Conclusion}

Our simulations show that small-scale vortex tubes representing whirlpool-type motions at intersections of intergranular lanes may play important roles in dynamics of the quiet-Sun and magnetic regions. The results indicate that the process of spontaneous formation of small-scale magnetic structures and their accumulation into a largescale magnetic structure is associated with strong vortical downdrafts developed around these structures. The resulting stable pore-like magnetic structure has the highest field strength of $\sim 6 \mathrm{kG}$ at the depth of $1-4 \mathrm{Mm}$ and $\sim 1.5 \mathrm{kG}$ at the surface (Kitiashvili et al. 2010). It has a cluster-like internal structurization, and seems to be maintained by strong downdrafts converging around this structure and extending into the deep layers. The simulations show that this internal dynamics plays a critical role in the magnetic self-organization of solar magnetic fields and formation of large-scale magnetic structures.

\section{Acknowledgement}

The presentation of this paper at the IAU Symposium 273 was possible due to the support from the National Science Foundation: grant numbers ATM 0548260, AST 0968672, and the NASA - Living With a Star grant, number 09-LWSTRT09-0039. Also, we thank the International Space Science Institute (Bern) for the opportunity to discuss these results at the international team meeting on solar magnetism.

\section{References}

Bonet, J. A., Márquez, I., Sánchez Almeida, J., Cabello, I. \& Domingo, V., 2008, Astrophys. J., 687, L131 
Bonet, J. A., Márquez, I., Sánchez Almeida, J., Palacios, J. et al., 2010, arXiv:1009.1992

Cheung, M. C. M., Schüssler, M., Tarbell, T. D. \& Title, A. M., 2008, Astrophys. J., 687, 1373

Jacoutot, L., Kosovichev, A. G., Wray, A. A., \& Mansour, N. N., 2008, Astrophys. J., 684, L51

Kitiashvili, I. N., Kosovichev, A. G., Wray, A. A., \& Mansour, N. N., 2009, Astrophys. J., 700, L178

Kitiashvili, I. N., Kosovichev, A. G., Wray, A. A., \& Mansour, N. N., 2010, Astrophys. J., 719, 307.

Pötzi, W. \& Brandt, P. N., 2005, Hvar Obs. Bull., 29, 61.

Rempel, M., Schüssler, M., \& Knölker, M., 2009, Astrophys. J., 691, 640

Stein, R. F. \& Nordlund, Å., 2001, Astrophys. J., 546, 585.

Stein, R. F., Bercik, D. \& Nordlund, Å., 2003, ASP Conf. Ser., 286, 121

Stein, R. F., Lagerfjärd, A., Nordlund, Å., \& Georgobiani, D., 2010, Solar Phys. (in press) 